\title{
Congenital obstructive bowel anomalies presenting after neonatal age
}

\section{Sebastian O Ekenze, Emmanuel I Nwangwu, Uchechukwu O Ezomike}

Date Received: 17-Jun-2018

Revision Received: 24-Jul-2018

Date Accepted: 11-Aug-2018

Correspondence:

sebekenze@gmail.com

Sub-Department of Paediatric Surgery, College of Medicine, University of Nigeria, Enugu Campus

\begin{abstract}
Background
Abstract

Delayed presentation might affect the ultimate management of children with congenital bowel obstructive bowel anomalies (CBA). We evaluated the profile, challenges of treatment and outcome of CBA presenting after neonatal age.

Methods

We did a retrospective analysis of data of children with CBA presenting after neonatal age from January 2013 to November 2017. We used the Statistical Package for Social Sciences (SPSS) for data entry and analysis.

Results

There were 57 cases in total comprising of Hirschsprung's disease (HD; 37 cases), anorectal malformation (ARM; 15 cases), and duodenojejunal web (5 cases), with median age of 9 months (IQR 4 months -2 years) on presentation. Overall, $52(91.2 \%)$ patients had one or more complications on presentation. Definitive procedure did not differ from established operations, but only $9(15.8 \%)$ had primary procedures and $48(84.2 \%)$ cases required multi-stage treatment. After an average follow up period of 19.5 months (range: $1-45$ months), 18 (31.6\%) cases developed procedure-related complications and $3(5.3 \%)$ had residual bowel dysfunction, but there was no mortality. The morbidity was limited to cases with HD and ARM.

Conclusion

In our setting, HD is the commonest bowel anomaly that presents after the neonatal age. The delayed presentation may predispose to complications and preclude single-stage treatment in some cases. Training of healthcare providers to improve recognition and early referral of these anomalies may lead to early diagnosis and minimize morbidity.
\end{abstract}

Keywords: congenital bowel anomaly, delayed presentation; challenges; outcome; developing country

\section{Introduction}

Congenital obstructive anomalies of the bowel can involve any part of the bowel from the duodenum to the anal canal. The nature of the anomalies ranges from complete atresia and varying degrees of stenosis, to functional obstruction due to defect of neuroregulatory components ${ }^{1}$. These may be structural anomalies like anorectal malformations, intestinal atresias and duplications or functional anomalies as in cases of Hirschsprung's disease. The presentations of these disorders vary significantly and depend on the severity of the disease as well as the part of the bowel involved ${ }^{1-3}$. It is expected that these disorders will manifest during the neonatal period and with available clinical skills, diagnosis should be made during this period. However, studies ${ }^{2-5}$ have shown that for varying reasons, a considerable number of these cases present later during infancy or even late childhood. Such delayed presentation might affect the ultimate management of these patients and may provide added structural, functional and psychological morbidity ${ }^{4,5}$. There are indications that most of these delayed presentations occur in the developing world where the situation is also compounded by lack of facilities and dearth of trained personnel ${ }^{4,6-8}$. An evaluation of these late-presenting anomalies may provide valuable data on the anomalies and the challenges of their treatment. Ultimately, such data will engender development of feasible initiatives to ensure timely diagnosis and workable solutions to the challenges of treatment. This study looks at the profile, management challenges and outcome of congenital obstructive bowel anomalies presenting after neonatal age.

\section{Methods}

At the University of Nigeria Teaching Hospital, and Mother of Christ Specialist Hospital, Enugu, the paediatric surgical unit undertakes the management of children presenting with congenital obstructing bowel anomalies. From January 2013, we commenced data collection on cases presenting after the neonatal age. This study is a retrospective analysis of data collected till November 2017. On presentation, patients were clinically evaluated and resuscitated as needed. Further treatment depended on the findings of imaging and blood investigations. For children with Hirschsprung's disease, the presence of enterocolitis or absolute obstruction that did not respond to enema or washout, or those with massively dilated colon, we undertook colostomy for adequate bowel decompression. For the rest of the Hirschsprung's cases non-operative decompression was carried out. Definitive operation was carried out when the bowel was adequately decompressed, and enterocolitis, if present, was adequately 
treated. In patients with anorectalTable 1: The mean age on presentation and presentation type for the disorders among the 57 malformation, colostomy was carriedpatients with congenital bowel anomalies

out if there was cloacal anomaly or when vestibular fistula did no decompress adequately. Definitive operation was done when the large bowel was adequately decompressed, and in the case of cloacal anomaly, the anatomy was well defined. For small bowel web, we undertook primary repair after adequate resuscitation. In the course of evaluation and treatment of these cases, the following data were collected: age on presentation, sex, main presentation and finding on physical examination, main finding on investigation, state Diagnosis of the patient on presentation, and reason for delayed presentation. The other data collected were diagnosis, initial treatment, definitive treatment interval from initial treatment to definitive treatment, complications of treatment, outcome, and follow up duration.

We used the Statistical Package for Social Sciences (SPSS 17.0 version, SPSS Inc, Chicago, Ill) for data entry and analysis. Results were expressed as percentages, or mean, or median with inter-quartile range (IQR).

\section{Results}

There were a total of 57 patients comprising of 35 male (61.4\%) and 22 female (38.6\%). The overall median age on presentation was 9 months (range: 6 weeks -11 years; IQR: 4 months -2 years). Of these patients, 6 were born in the two centres and the remaining 51 patients were born outside these centres.

\section{Presentation}

Of the 57 cases, $37(64.9 \%)$ presented with Hirschsprung's disease (HD), $15(26.3 \%)$ had anorectal malformation (ARM), 4 (7.0\%) had duodenal web, and one patient (1.8\%) had jejunal web.

Hirschsprung's disease: The mean age at presentation was 2.4 years. The youngest age among the cohort of patients with HD was 3 months in total colonic HD, and the oldest age was 11 years in a patient with rectosigmoid disease. In these patients, aganglinosis was at rectosigmoid (27), descending colon (7), total colonic (2) and one anal canal (ultrashort segment). A total of 21 patients presented with massive bowel dilatation, 8 had HD-related enterocolitis, and 6 had absolute obstruction.

Anorectal malformations: In this, there were rectovestibular (7), cloaca (4), perineal fistula (2) and one case each of anovestibular and rectal atresia. The oldest presentation in ARM was 10 years in a female with perineal fistula, and the youngest was a 6 -weeks old with vestibular fistula. The mean age on presentation was 12 months. Overall, 8 cases had severe difficulty with stooling through the fistula, and $5 \mathrm{had}$ chronic urinary tract infection. $\mathrm{n} \quad$ Mean age at presentation (Range) Presentation

Elective Emergency

2.4 years ( 3 months -11 years)
1-year ( 6 weeks -10 years)
9 months ( 6 weeks -2 years)
4 months

$25(67.6 \%)$

$13(86.7 \%)$

$0(0 \%)$

$1(100 \%)$

$12(32.4 \%)$

$2(13.3 \%)$

$4(100 \%)$

procedure prior to the

$\begin{array}{llllllll}\text { Hirschsprung's disease } & 21 & 8 & 6 & - & - & 33 & 2 \\ \text { Anorectal malformation } & - & - & - & 8 & 5 & 13 & \end{array}$

* MBD (Massive bowel dilatation); IO (Intractable obstruction); SDS (Severe

difficulty with stooling); CUC (Chronic urinary contamination)

Small bowel web: The mean age at presentation for the 4 patients with duodenal web was 9 months (range: 6 weeks -2 years). All the cases had web at the second part of the duodenum. The patient with jejunal web presented at the age of 4 months. All the 4 cases of duodenal web presented with failure to thrive, dehydration and electrolyte derangement. Overall, 18 (31.6\%) presented as emergency, and 39 (68.4\%) presented as elective (Table 1$)$. The main reason for delayed presentation was delayed referral in 31 (54.4\%) of the patients, while in $26(45.6 \%)$ cases, the caregiver did notice that there was problem prior to presentation. Only $8(14 \%)$ cases reported having a well child examination.

\section{Treatment}

On initial evaluation, a total of $52(91.2 \%)$ patients had one or more complications of the disease and required either fluid and electrolyte resuscitation or diverting stoma, or both prior to definitive procedure. A total of 35 cases of $\mathrm{HD}$ required initial diverting stoma (colostomy 33; ileostomy 2) for massive bowel dilatation $(n=21)$, HD related enterocolitis $(n=8)$, and absolute obstruction $(n=6)$. In ARM, the 8 cases that had severe difficulty with stooling, and 5 that had chronic urinary tract contamination had initial diverting colostomy (Table 2).

All the 4 cases of duodenal web had fluid and electrolyte derangement requiring fluid and electrolyte resuscitation. Definitive operative treatment was undertaken at the median age of 2 years (range: 6 weeks - 13 years; IQR: 14 months -5 years). The distribution of the definitive operative treatment and the mean age at which the procedures were undertaken for the various disorders is shown in table 3 . 
Table 3: The distribution of the definitive operative treatment and the mean age at which the procedures were undertaken for the various disorders among the 57 children with congenital bowel anomalies presenting after neonatal age

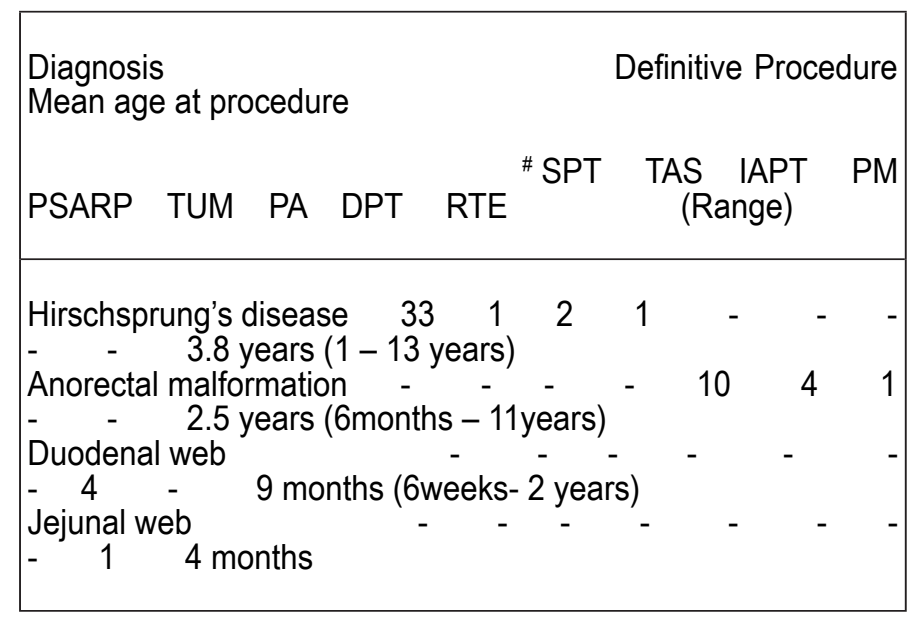

SPT (Swenson Pullthough); TAS (Transanal Swenson Pullthough); IAPT (Total colectomy + ileoanal pullthrough); PM (posterior myectomy); PSARP (Posterior sagittal anorectoplasty); TUM (Total urogenital mobilization); PA (Perineal anoplasty); DPT (Resection of duodenal web and duodenoplasty); RTE (Resection, tapering enteroplasty and end to end anastomosis)

\section{Outcomes}

There were $18(31.6 \%)$ patients with procedure-related complications. The complications were Soiling/bowel accidents (5), stoma prolapse (3), anastomotic leak (2), enterocolitis (2), skin excoriation (2), superficial wound dehiscence (2), and one case each of anastomotic stenosis and anal stenosis. The complications were limited to cases of HD $(n=13)$ and ARM $(n=5)$. The two cases with anastomotic leak required re-colostomy and the other complications were managed non-operatively. Overall, there was no mortality, but $3(5.2 \%)$ patients ( 2 cases of HD and 1case of ARM) had residual soiling/bowel accidents. The mean duration of follow up was 19.5 months ( $1-45$ months).

\section{Discussion}

This study has shown that Hirschsprung's disease (HD) is the commonest congenital obstructive bowel anomaly that might present for treatment after the neonatal period in our setting. A similar finding has been highlighted in previous studies from some other developing countries ${ }^{5,8,9}$. The other anomalies in the present report viz- anorectal malformation with rectovestibular fistula, and duodenal and jejunal web, have also been previously reported as presenting occasionally in the post-neonatal period ${ }^{5-7,10}$. It may be reasonable to assume that these anomalies might be of the mild or moderate severity types. It was noted that mild to moderate HD and ARM in girls with low fistula presented late as they are not symptomatic early, and some duodenal and jejunal webs, as in our patient cohort, are incomplete webs; hence, they have delayed presentation ${ }^{6,7,10}$. However, the presentation of cases of total colonic HD after the neonatal period in our series and the report of high anorectal malformation in male in previous studies ${ }^{5,711}$ might indicate that some severe forms of these anomalies may also be involved. In all these cases, the anomalies may have allowed some function either as decompression of the large bowel or allowing passage of luminal content via a hole in the small bowel web past the neonatal age.

As a result of the well-established clinical features of these anomalies, it is expected that they would be identified and evaluation and treatment commenced at birth or shortly thereafter. Our study and some previous reports ${ }^{4-12}$ have shown that timely diagnosis of these anomalies may not be achievable at all times. The putative reason for delayed diagnosis of these disorders in our setting may not be conclusively deduced from the present report. Howbeit, some of our findings might point to some contributory factors like delayed detection or delayed referral or both. Delayed detection may arise from inadequate antenatal care and lack of routine well baby examination which has also been previously reported from some developing countries ${ }^{13,14}$; or inability of the healthcare personnel at the initial point of contact of the patients to identify and evaluate for these disorders. Published works from some developing countries ${ }^{4,5,7-9,13}$ have also highlighted the latter reason for delayed detection, indicating that it might contribute to most of the delays in definitive diagnosis and treatment; delayed referral is a consequence of delayed detection. Another important factor in delayed management is the paediatric surgery work force shortage in most parts of Africa. The number of paediatric surgeons in Nigeria and most subSaharan African countries is small and this is compounded by a lack of paediatric surgeons outside teaching and tertiary hospitals ${ }^{15}$. It is estimated that the number of paediatric surgeons in sub-Saharan Africa ranges from 0.06 to 2.2 per million population ${ }^{16,17}$. The net effect is a considerable limitation in the diagnosis and care of these children with congenital anomalies. To improve time to diagnosis, it may be expedient to improve antenatal care and include well baby examination as part of compulsory procedures before postnatal discharge of newborn. In addition, there may be a need to organize regular training and retraining of healthcare providers in the primary and secondary care centres to recognize features of these disorders and the need for early referral. In addition, paediatric surgery capacity may need to be scaled up in our setting.

In the present report, about a third of the cases presented as emergency though more than $90 \%$ had one or more complications of the disease on presentation. The mean age on presentation for each of the anomalies might explain the high rate of complication of the diseases on presentation. Among the cases of HD, anorectal malformation, and small bowel web previously reported as presenting after the neonatal age, complications of the disease were noted in more than $50 \%$ of the patients ${ }^{4,5-7,9-11,18}$. The reported complications such as HD-related enterocolitis, massively dilated colon, absolute obstruction in HD, poor decompression and chronic urinary tract infection in ARM, failure to thrive, and electrolyte derangement in small bowel web were also noted in the present report. The high incidence of these complications on presentation implies that surgeons managing such cases may have to develop strategies to address these problems in order to minimize morbidity and mortality. These complications have been shown to affect the treatment of the anomalies, ${ }^{4,}$. 
In cases of large bowel anomalies with chronically dilated bowel, it may be difficult to perform a single-stage procedure. Previous studies indicate that non-operative decompression of the bowel may not be feasible in more than $50 \%$ of the affected cases and these might require colostomy $y^{4,9,11,12}$. This failure may be related to a high incidence of impacted feces and, possibly, associated chronic bowel inflammation ${ }^{4,9,18}$. This may compel the use of multi-stage technique of initial colostomy, definitive procedure, and colostomy closure instead of the current trend of primary pull-through for the rectosigmoid, descending colon or ultra-short $\mathrm{HD}$, and primary posterior sagittal anorectoplasty (PSARP) for ARM with rectovestibular fistula ${ }^{19,20}$. The use of multistage has been known to increase the susceptibility for postoperative complications $s^{4,9}$. In cases of small bowel web, the failure to thrive and electrolyte derangement provide management challenges especially in a developing country setting with lack of facilities for parenteral nutrition and monitoring, and may call for a more aggressive resuscitation ${ }^{6,10}$. However, the operative procedure is not different from that applied in cases presenting earlier.

The overall outcome of treatment in the present report may be similar to results reported in studies involving younger children ${ }^{2,19,21}$. However, for the reasons already stated, the incidence of complications of treatment may be higher in older children than in younger children. Mortality is rare ${ }^{4,9,12}$. Given the short duration of follow-up in the present study, the overall complications may increase with the occurrence of some late complications as the duration of follow-up increases.

\section{Limitations of the study}

This study was limited by the small number of cases and the design that did not include data on the mode of delivery of the patients, the treatment given before presentation to the centres, and the outcome of management of patients that presented during the neonatal period. This precluded a more detailed analysis. The other limitation is the reliance on tertiary-centre patients that excluded a sizable number of cases that could have presented at the secondary or other facilities.

\section{Conclusion}

In our setting, Hirschsprung's disease, anorectal malformation, and duodenal and jejunal web are the obstructing bowel anomalies that might present for treatment after the neonatal period. A considerable number of cases were not given well child examination, but the main contributor to late presentation was delayed referral. A substantial proportion of these patients had complication of the disease on presentation, necessitating that some cases of large bowel anomalies that would have been managed with single procedure with early diagnosis may require multistage procedure. There may be no mortality but treatmentrelated complications might be higher than cases identified and managed from neonatal period. Emphasizing well baby examination and education of healthcare personnel at the primary and secondary health facilities on early identification and referral of these disorders might improve time to diagnosis and overall outcome.

\section{Conflict of interest}

The authors declare that they have no conflict of interest.

\section{References}

1. Hajivassiliou CA. Intestinal obstruction in neonates/pediatric surgery. Semin Pediatr Surg. 2003; 12 (4): 241 - 253

2. Morris G, Kennedy A Jr, Cochran W. Small bowel congenital Anomalies: A review and update. Curr Gastroenterol Rep. 2016; 18(4):16. doi: 10.1007/s11894-016-0490-4.

3. Wetherill C, Sutcliffe J. Hirschsprung disease and anorectal malformation. Early Hum Dev. 2014; 90(12): 927-932. doi: 10.1016/j. earlhumdev.2014.09.016.

4. Ekenze SO, Ngaikedi C, Obasi AA. Problems and outcome of Hirschsprung's disease presenting after 1 year of age in a developing country. World J Surg. 2011; 35 (1): 22 - 26. doi:10.1007/s00268-0100828-2

5. Vaos G, Misiakos E. Congenital anomalies of the gastrointestinal tract diagnosed in Adulthood - diagnosis and management. J Gastrointest Surg. 2010; 14(5): 916-925. doi: 10.1007/s11605-009-1124-z.

6. AIGhannam R, Yousef YA. Delayed presentation of a duodenal web. J Pediatr Surg Case Reports. 2015; 3(12): 530 - 533. doi:/10.1016/j. epsc. 2015.10 .014

7. Sharma S, Gupta DK. Delayed presentation of anorectal malformation for definitive surgery. Pediatr Surg Int. 2012; 28(8): 831-834. doi: 10.1007/s00383-012-3128-0.

8. Bandré E1, Kaboré RA, Ouedraogo I, Soré O, Tapsoba T, Bambara $\mathrm{C}$, et al. Hirschsprung's disease: management problem in a developing country. Afr J Paediatr Surg. 2010; 7(3): 166-8. doi: 10.4103/01896725.70418 .

9. Sharma S, Gupta DK. Hirschsprung's disease presenting beyond infancy: surgical options and postoperative outcome. Pediatr Surg Int. 2012; 28 (1):5-8. doi: 10.1007/s00383-011-3002-5.

10. Sarkar S, Apte A, Sarkar N, Sarkar D, Longia S. Vomiting and food refusal causing failure to thrive in a 2 year old: An unusual and late manifestation of congenital duodenal web. BMJ Case Rep 2011; doi: 10.1136/bcr.01.2011.3779

11. Pandey A, Gangopadhyay AN, Kumar V, Sharma SP. High anorectal malformation in a five-month-old boy: A case report. J Med Case Reports. 2010; 4: 296. doi: 10.1186/1752-1947-4-296

12. Chakravartty S, Maity K, Ghosh D, Choudhury CR, Das S. Successful management in neglected cases of adult anorectal malformation. Singapore Med J. 2009; 50(8): e280-2.

13. Ekenze SO, Ajuzieogu OV, Nwomeh BC. Challenges of management and outcome of neonatal surgery in Africa: A systematic review. Pediatr Surg Int. 2016; 32 (3): 291 - 299. doi:10.1007/s00383-016-3861-x 14. Garces AL, McClure EM, Pérez W, Hambidge KM, Krebs NF, Figueroa L, et al. The global network neonatal cause of death for lowresource settings. Acta Paediatricica. 2017; 106 (6): 904 - 911. doi:

10.1111/apa.13805

15. Elhalaby EA, Uba FA, Borgstein ES, Rode H, Millar AJ.

Training and practice of paediatric surgery in Africa: Past, present, and future. Semin Pediatr Surg. 2012; 21:103-110. doi: 10.1053/j. sempedsurg.2012.01.002.

16. Chirdan LB, Ameh EA, Abantanga FA, Sidler D, Elhalaby EA. Challenges of training and delivery of pediatric surgical services in Africa. J Pediatr Surg. 2010; 45 (3):610-618. doi: 10.1016/j. jpedsurg.2009.11.007

17. Ameh EA, Seyi-Olajide JO, Sholadoye TT. Neonatal surgical care: A review of the burden, progress and challenges in sub-Saharan Africa. Pediatr Int Child Health. 2015; 35 (3) : 243 - 251. doi: 10.1179/2046905515Y.0000000033

18. Ekenze SO, Agugua-Obianyo NE, Amah CC. Colostomy for large bowel anomalies in children: A case controlled study. Int J Surg. 2007; 5 (4): 273 - 277. doi:10.1016/j.ijsu.2007.01.008

19. Bischoff A, Levitt MA, Peña A. Update on the management of anorectal malformations.Pediatr Surg Int. 2013; 29(9): 899-904. doi: 10.1007/s00383-013-3355-z.

20. Chung PHY, Wong KKY, Tam PKH, Leung MWY, Chao NSY, Liu $\mathrm{KKW}$, et al. et al. Are all patients with short segment Hirschsprung's disease equal? A retrospective multicenter study. Pediatr Surg Int. 2018; 
34 (1): 47 - 53. doi:10.1007/s00383-017-4202-4.

21. Higashi M, Ieiri S, Teshiba R, Saeki I, Taguchi T. Hirschsprung's disease patients diagnosed at over 15 years of age: An analysis of a
Japanese nationwide survey. Pediatr Surg Int. 2009; 25 (11) :945-947. doi: $10.1007 / \mathrm{s} 00383-009-2447-2$ 